Revolution leben wir in einer real existierenden globalen Weltgesellschaft. Die Nationalstaats-Verfassungen (nationalstaatliche GroB-Mächte) sind ein historisches Relikt. In Perspektive auf eine weltweite Civil Society, die föderativ und dezentral aufgebaut sein soll, müssen die Menschenrechte aus der Sicht von Frauen (mit einem menschenrechtlich begründeten >Right of Food<) erst noch geschrieben werden.

\title{
Anmerkungen
}

1 Schultz, Imngard; Weiland, Monika, Frauen und Müll - Frauen als Handelnde in der kommunalen Abfallwirtschaft, Frankfurt/M. 1991.

2 Fietkau, H.-J.; Kessel, H.: UmweltbewuBtsein - nur ein Schlagwort? In: W. Beer, G. de Haan (Hg.), Ökopädagogik, Weinheim 1984.

Doris Janshen

\section{Bestimmung über Vor-Krieg, Krieg und Frieden}

Unsere derzeitige Verfassung enthält nicht nur Grundsätze und Konfliktregelungen für sogenannte Friedenszeiten, auch das Thema Krieg oder - wie es so sprachtückisch heißt - der »Verteidigungsfall« ist kursorisch eingeblendet. Hier wird sichtbar, daß wir uns mitten im Frieden immer auch in einer Zeit der Vorbereitung des nächsten Krieges befinden, im Vorkrieg mithin.

Grundlage aller Absicherungen für den Fall eines Krieges ist der berühmte Art. 12a, der seit 1956 die Wehrpflicht gebietet. Art. 4 Abs. 3 setzt der Kriegsdienstverweigerung Grenzen. Art. 26 enthält das Verbot eines Angriffskrieges für die Bundesrepublik und Kapitel Xa unserer Verfassung enthält ab Art. 115 a weitere Rahmenbedingungen für den sogenannten Spannungs- und Verteidigungsfall. Fast alle dieser Festlegungen betreffen ausschlieBlich Männer. Nur in zwei Fällen sind Frauen erwähnt. Art. 12a verbietet für Frauen den Dienst mit der Waffe, während Art. 87a klassisch weibliche Hilfsdienste für den Kriegsfall fordert. Frauen sind beim Thema Krieg scheinbar kaum gefordert, da sie als kämpfendes Mitglied der Bundeswehr nicht vorgesehen sind. Offenbar hat dies auch Konsequenzen für die politische Orientierung in der Frauenbewegung. Zwar haben sich in der neuen deutschen Frauenbewegung der vergangenen Jahre viele Frauen gegen Waffen und für den Frieden engagiert, es hat jedoch so gut wie keine Klärung darüber stattgefunden, ob und inwieweit die demokratischen Grundrechte für Frauen noch greifen, wenn es um die Entscheidung über Krieg oder Frieden 
geht. Auch in der Auseinandersetzung von Frauen über und für eine neue Verfassung überwiegen Gedanken zum $\S 218$ und zu Varianten des Familienschutzes. Das Nachdenken über das Verhältmis von Frauen zum Krieg erzeugt dagegen Berührungsängste, wenn nicht gar Abwehr.

Wie in der geltenden Verfassung selbst äuBern sich auch in der gegenwärtigen frauenpolitischen Diskussion Geschlechtsstereotype, von denen eine ganze Reihe von Wissenschaftlerinnen und Politikerinnen meinen, nicht mehr darüber reden zu müssen. Männer behaupten für sich das Geschlechtsprivileg des Tötens, während Frauen sich auf das Geschlechtsprivileg des Gebärens und seiner Rahmenbedingungen konzentrieren. Heide Hering hat in ihrem Beitrag deutlich darauf hingewiesen, daß sich die Differenz zum männlichen Geschlecht aus dem Privileg der Geburt herleite. Schaue ich in die fast ausschlieBlich von Männern verfaBte Literatur zum Militär hinein, dann wird mir in unzähligen Beispielen vorgeführt, wie sehr die gesellschaftlich legitimierte Form des Tötens im Militär Männlichkeit bestätigt und reproduziert, mit anderen Worten: den Geschlechtsstolz stereotyper Männlichkeit erhält. Ich fürchte, daß alle Erfolge der Frauenpolitik bezüglich der Geschlechterpolarität im zivilen Sektor vorläufig bleiben werden, solange der unzivile Sektor - das Militär - immer wieder den stereotypen Gegensatz von Töten und Gebären reproduziert.

Es kann gar nicht unterschätzt werden, was es für die Sozialisation von Männern und die Reproduktion traditioneller Männlichkeit bedeutet, daB alljährlich junge Männer in einem noch prägefähigen Alter in die Armee einrücken. Hier findet nicht nur eine Einübung in Männerkumpanei statt, hier wird auch erlernt, wie nach dem Organisationsmuster einer totalen Institution gesellschaftliche Prozesse beherrschbar sind. Max Weber hat bereits darauf hingewiesen, daB militärische Ordnung und Disziplin eine wesentliche Grundlage sowohl für den bürokratischen Staat als auch für den modernen kapitalistischen Betrieb darstellen. Auch meine bisherigen Forschungen haben in geradezu frappierender Weise bestätigt, wie sehr militärische Handlungsnormen die zivile Karriere des Mannes fundieren. Mit anderen Worten: frauenfremde und frauenfeindliche Strukturen im Erwerbsbereich, die wir lange als bereichsspezifisch bekämpft haben, sind letztlich der strukturbestimmenden Rolle des Militärs in der patriarchalen Gesellschaft geschuldet. Was den Männerstolz des einzelnen Individuums bestätigt - das Privileg der Waffe -, ist in Kollektive geweitet Garant für das Selbstbewußtsein des patriarchalen Staates. Souverän sei der Staat erst mit einer Armee, hieß es vor der Wiederbewaffnung der Bundesrepublik in den 50er Jahren. Auch in der gegenwärtigen Auseinandersetzung über den Einsatz der Bundeswehr außerhalb der NATO fällt immer wieder der Begriff der vollen Souveränität. Die Selbstachtung des Staates ist auf junge Männer angewiesen, die die gesellschaftlich legitimierte Form des Tötens erlernt haben.

Vor dem Hintergrund der - hier nur kurz gefaßten Analyse - komme ich zu folgenden Vorschlägen für eine andere Gesellschaft, sprich auch für eine neue Verfassung:

1. Töten ist mit der Würde eines jeden Menschen unvereinbar. Kein Mensch darf zur Arbeit mit der Waffe gezwungen werden. Gesellschaft und Staat verpflich- 
ten sich auf die radikale Konversion des militärisch-technischen Sektors. Nachfolgende Gesetze regeln die Kontrolle dieses Prozesses.

Erläuterung: Die Formulierung reflektiert die gegenwärtige Absurdität, daß es ein Geschlecht auszeichnet, das Geschäft des Tötens zu erlernen, während es mit der Würde des anderen nicht vereinbar ist, sich hier einzumischen. Doch die Würde des Menschen ist auch geschlechtsspezifisch nicht teilbar. Ohne Angabe von Gründen kann sich jeder Mensch der Arbeit mit der Waffe verweigern. Die Wiederbewaffnung der Bundesrepublik war schneller zu bewerkstelligen als die nun intendierte Abschaffung der Bundeswehr und aller Nachfolgeeinrichtungen. Mit verhaltenem Optimismus ist daher nur von einer mradikalen Konversion des militärisch-technischen Sektors« die Rede. Die Versuche der Gewerkschaften haben in den vergangenen Jahren gezeigt, wie schwierig es ist, Rüstungstechnik in zivile Projekte zu konvertieren. In der hier nun projektierten Veränderung geht es jedoch nicht nur um die Umgestaltung von Fertigungsstraßen, sondern um die Neugestaltung unserer wirtschaftlichen Ordnung. Veränderung wird daher als kontrollierter ProzeB gesehen.

2. Frauen werden in allen Entscheidungsgremien des Militärs sowie militärbezogenen Gremien der Politik in einem Maße kooptiert, das ihrem Anteil an der Bevölkerung entspricht. Für diese Kooptation ist eine militärische Ausbildung keine Voraussetzung.

Erläuterung: Dieser Vorschlag geht aus von den jetzt geltenden Regelungen für den Spannungs- bzw. Verteidigungsfall. Er gilt jedoch nicht nur für diese Zeiten, sondern generell. Unsere Verfassung sieht vor, daB Bundestag und Bundesrat mit einer Zweidrittelmehrheit beschließen, daB der Verteidigungsfall eingetreten ist. Dem Kanzler wird damit die Oberhoheit über die Streitkräfte eingeräumt. Stellen wir uns nun vor, wie überzeugend hoch der Anteil von Frauen im Bundestag ist, und bemühen wir weiter unsere Phantasie, wer in einer solchen Situation die Beratungsgremien des Kanzlers bevölkern wird. Das sind hohe Militärs, Vertreter aus der rüstungstechnischen Industrie und Berater aus dem Verteidigungsministerium. Der männliche Plural ist hier angebracht, denn Frauen sind in diesen Bereichen in entschiedener Vereinzelung.

$\mathrm{Da}$ ich mich Petra Kellys Auffassung nicht anschließen kann, daß die Nichtpräsenz von Frauen im Militär Kriege verhindere - wenn ich mich umschaue, kann ich es einfach nicht glauben -, muB ich fragen, wie Frauen zu angemessenen Mitbestimmungsrechten kommen: über Krieg und Frieden, über Leben und Tod. Dabei halte ich es fuir unerheblich, ob einzelne Frauen im Militärbereich aktiv mitwirken. Ihre Mitwirkung in einer so hochkarätig patriarchalen Institution ist für unsere politischen Ziele unerheblich. Entscheidend ist die Frage, ob und wie Frauen über Krieg und Frieden mitbestimmen können, ohne den »Dienst« mit der Waffe erlernt zu haben. Wie die Geschichte zeigt, reicht militärische Kompetenz nicht aus, um Kriege zu verhindern. Im sogenannten Spannungsfall wird jedoch nur nach militärischer Kompetenz gefragt. Warum entscheidet hier nicht soziale Kompetenz? Soziale Kompetenz wird Frauen in der patriarchalen 
Gesellschaft aufgezwungen und abverlangt. Warum entscheiden diese Fähigkeiten nicht über Krieg und Frieden, über Prioritäten bei technischen Investitionen? Warum sind sie in diese Entscheidungsprozesse nicht immer eingebunden? Nicht nur in Zeiten des Krieges, sondern auch des Vor-Krieges? Mein Vorschlag sucht nach demokratischen Mitwirkungsrechten für Frauen unabhängig von der aktuellen politischen Situation und unabhängig von der Einübung in die "Waffenkunst «.

3. Frauen und Männern steht die Möglichkeit der Kriegsverweigerung aus Überzeugung offen, solange der militärische Sektor nicht gänzlich in Einrichtungen für sozial förderliche Zwecke transformiert wurde.

Erläuterung: Dieser Vorschlag resultiert aus der Reflexion des gegenwärtigen Art. 4 Abs. 3. Anders als in diesem sind Frauen und Männer angesprochen. Männer haben derzeit als Kriegsdienstverweigerer die Möglichkeit, sich gegen militärische Gewalt auszusprechen. Sind es viele, für die Regierenden zu viele, dann werden sie zu Sand im Getriebe, d.h. zu einem diskussionswürdigen Faktor der politischen Diskussion. Frauen dagegen wird militärische Abstinenz von vornherein unterstellt. Wo sie sich dem Militär und angelagerten Bereichen fernhalten, wird dies nicht als Widerstand angesehen, sondern als eine Variante geschlechtsspezifischer Eigenart. Damit wird ein Demokratiedefizit sichtbar. Da Frauen den militärischen Sektor nicht aktiv, sondern allenfalls durch ihre Subsistenzarbeiten stützen, wird ihr politischer Widerstand nicht als ein solcher anerkannt und verpufft wirkungslos. Deshalb ist in diesem Vorschlag nicht von Kriegsdienstverweigerung, sondern von Kriegsverweigerung die Rede. Alle Tätigkeiten, die der Kriegsvorbereitung bzw. der Kriegsführung dienen, sind verweigerungsfähig. Damit aber sind alle Handlungen und Arbeiten angesprochen, die der Fundierung kriegerischer Aktivitäten dienen. Ein Beispiel: die Montiererin in der Rüstungsindustrie könnte vor diesem Hintergrund auf einen zivilen Arbeitsplatz klagen. Bedeutsam in diesem Kontext ist auch der Ersatz des Wortes "Gewissen« durch das Wort »Überzeugung«. Es geht nicht mehr um den individuellen Nachweis, aus Gewissensgründen auch in extrem bedrohlichen Situationen nicht zur Gewalt zu greifen, sondern um die politische Einsicht, daB Kriege kein Mittel zu internationaler Konfliktregelung sind. Die Motivation von Frauen, auf die elementaren Regelungen zu Vor-Krieg und Krieg Einfluß zu nehmen, sind demnach mindestens doppelt stimuliert. Zum ersten insistieren wir auf dem Recht der Mitbestimmung über Krieg und Frieden. Zum zweiten aber geht es um einen ubiquitären Widerstand gegen den Kraftquell des Patriarchats: das Militär. 